\title{
SMART E-COMMERCE APPLICATION WITH SECURE BLOCKCHAIN
}

\author{
DR. B. VEERA JYOTHI ${ }^{1}$, DR. RAHUL ${ }^{2} \&$ V. JAIPAL REDDY ${ }^{3}$ \\ ${ }^{1}$ Department of IT, Chaitanya Bharathi Institute of Technology, 500075, India \\ ${ }^{2,3}$ Department of Mechanical Engineering, Chaitanya Bharathi Institute of Technology, 500075, India
}

\begin{abstract}
Now as technology is increasing there are lots and lots of developments in the field of science and technology but even in these days the credit/debit card information that we give while doing payment are getting stolen in some cases which is resulting in huge loss. But if the payments are digitalized where just by scanning QR code we can unlock the digital content like videos or any other items by using blockchain technology in which each transaction that is made in stored in blockchain and becomes immutable by making the chances of amount getting stolen to almost negligible. I would like to create a electronic commerce website containing videos which are locked and can only be accessed after the payment, a mobile wallet application for making payments and also create a user interface for blockchain which helps in tracking the transaction. By using this system the items can be purchased with safety, speed and accuracy. Also the whole transaction can be tracked in the blockchain.

KEYWORDS: Electronic Commerce, Cryptography, Data Security, Transaction Databases, Cryptographic Protocols
\end{abstract}

Received: Jun 09, 2020; Accepted: Jun 29, 2020; Published: Aug 07, 2020; Paper Id.: IJMPERDJUN2020682

\section{INTRODUCTION}

In normal payment methods that are used in e-commerce [1] site like debit/credit cards sometimes get saved as shown in Fig. 1 in the website which hackers can take advantage of steal the money. This will lead to huge loss and sometimes it is almost impossible to catch the hacker. Even the ATM the card pin may be secretly seen by someone using cameras.

\begin{tabular}{|c|c|}
\hline \multicolumn{2}{|l|}{ Payment Information } \\
\hline \multicolumn{2}{|l|}{ Credit Card (saved) } \\
\hline Credit Card Type: & MasterCard \\
\hline Credit Card Number: & 55555555555554444 \\
\hline Name on the Card: & EL HASSAN MATAR \\
\hline Expiration Date: & $12 / 2016$ \\
\hline \multicolumn{2}{|c|}{ Card Verification Value: 123} \\
\hline \multicolumn{2}{|c|}{ Order was placed using USD } \\
\hline
\end{tabular}

Figure 1: Credit/Debit Card Saved Information.

New research reveals the ease with which criminals can hack an account or working out the card number, expiry date, security code of any visa card or debit card can take as little as six seconds and uses nothing more than guesswork. Research published by IEEE security and privacy, shows how the so-called distributed guessing attack is able to circumvent all the security features put in a place to protect online payment [2].

The credit/debit card information that are given while doing payment are getting stolen in some cases which is resulting in huge loss. Exposing the flaws in VISA payment system [3], the team from the Newcastle 
University UK, found neither the network nor the banks were able to detect attackers making multiple, invalid attempts to get payment card data. By automatically and systematically generating different variations of the cards security data and firing it at multiple websites, within seconds hackers are able to get a 'hit' and verify all the necessary security data. Also high level payment services such as Apple Pay [4] and Android Pay [5] which based on visa are facing attacks .But if the payments are digitalized where just by scanning QR code [6] we can unlock the digital content like videos or any other items by using blockchain technology in which each transaction that is made in stored in blockchain and becomes immutable by making the chances of amount getting stolen to almost negligible [7]. E-commerce and blockchain [8] should be combined in order to overcome the security issues [9].

\section{Applications}

The payment systems can be revolutionized as the QR code based payments are used which ensures that transactions are instant [11] and accurate. Also blockchain is used along with the QR code based system so all the transactions are also provided high level security by storing them in blockchain. Once the transactions are stored in blockchain they become immutable which means no one can hack or change them. This payment system can be used in e-commerce site where large amount of transactions are done so that payments can be done with speed and security. It can help in making the banking sector better by reducing the chances of hacking accounts and also there will be no need of carrying any kind of credit/debit cards, just a blockchain based e-wallet [12] will be sufficient. It can also help in improving the supply chain management [13].

\section{METHODOLOGY}

As the credit/debit cards have many drawbacks so instead of this QR code based blockchain system can be used by which security and privacy can be increased. In this project the blockchain technology is used along with asp.net core and c\# usingwhich the payments can be made with qr code and each and every transaction can be traced in the blockchain. First we start with the blockchain user interface creation and it followed by a fully functionable blockchain web application where transactions are stored inside the blockchain. Then we create a ecommerce site which will consists of digital content which is locked by using qr code and it can be unlocked by paying some amount of coins. Then the e-commerce payment system is linked with the blockchain so that the security enhances. Then it is followed by a mobile wallet creation using Xamarin using which the payments are made.

An initial stage of building blockchain client application involves several tasks like public/private key generation which are basically used to uniquely identify a user, implementing wallet and web application. Transaction viewing page is created so as to track all the tasks taking place, and the implementation of consensus and miner, using longest chain rule which means who ever mines first will get the transaction into the block in order to avoid clashing inside the blockchain, overall flows etc. Followed by the ecommerce site in which we will implement signal $\mathrm{R}$ which is basically a library for asp.net that allows server code to send asynchronous notifications to client web applications. Then it follows by adding video unlocking page, or code generator page will be created and then implementation of payment API is done at last. Then in the mobile wallet development all the work related to the xml code will be done. At last all the projects will work together making it a complete project. Doing this the entire transactions will become transparent and even if there is any data breach the issues can be resolved within no time. Also there will be no need of saving the credit/debit cards or using them. The transactions using coins is way faster and secure compared to normal card payment method. This method can be implemented not only in ecommerce sites but also in banking sector, finance sector etc. Usage of the blockchain along with 
this platform adds more efficiency as blockchain is a decentralized distributed ledger [14] which does not have any central authority controlling it and also it is immutable which means once transaction or data entered cannot be modified.

The below figure represents the sequence diagram of customer named joe who tries to buy the digital content from the ecommerce site.

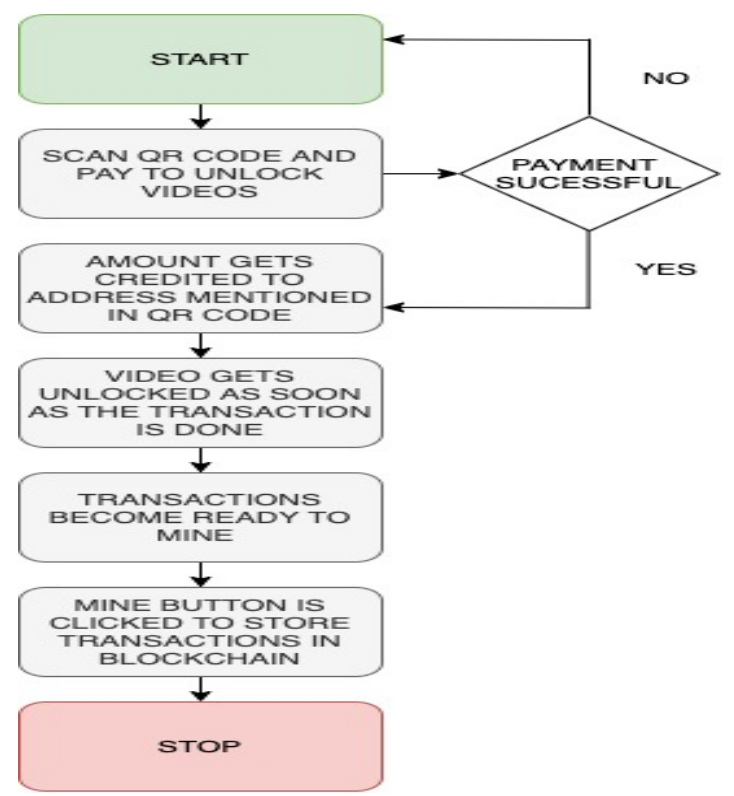

Figure 2: Flow Chart of the Proposed System.

As shown in Fig. 2 first we scan the QR code which has the sellers public address embedded in it and pay the amount mentioned on the locked video appropriately in order to unlock the video. If the payment is successful then the amount gets credited to the address mentioned in QR code and video gets unlocked immediately and becomes free to view. As soon as the video gets unlocked the transactions becomes ready to mine or ready to get added in blockchain. Once the mine button is clicked then the transaction gets stored in the blockchain and becomes immutable which means it can't be manipulated or changed.

\section{Architecture}

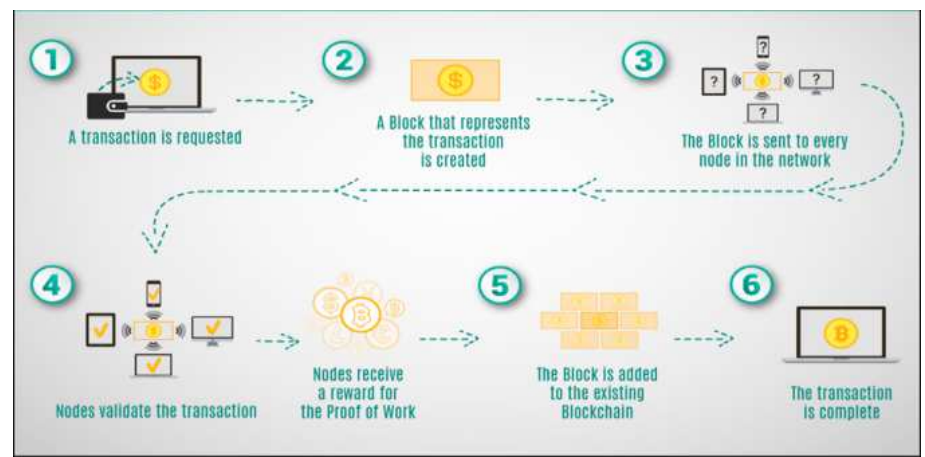

Figure 3: Blockchain Architecture.

The blockchain [15] involves certain process which needs to be carried out in order to complete the process as shown in Fig. 3 First the transaction is requested by user to the system then a block representing the transaction [16] is created now as the blockchain follows consensus [17] mechanism which is basically to the synchronize data among all the nodes present in the system the block is sent to every node in the network. The blockchain is also fault tolerant i.e. even if 
anyone tries to change one node rest all other nodes have also to be changed. As there is no central authority controlling the blockchain the nodes validate the transaction. Nodes receive a reward for the proof of work [18] which is basically the amount given to the miners. Then block is added to the existing blockchain by following the longest chain rule [19]. After completion of all these processes the transaction gets completed.

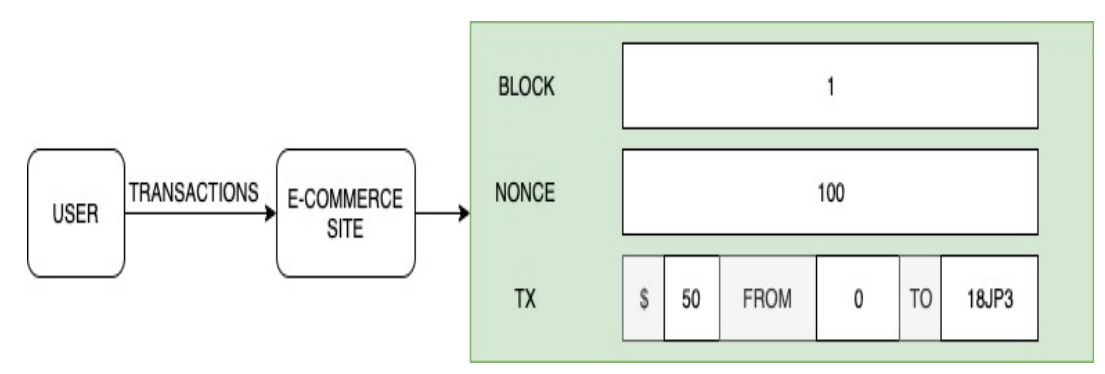

Figure 4: Project Architecture.

User scans the QR code and pays the required amount to unlock the video once the transactions are done they become ready to mine. Once the transactions are mined they are stored in blockchain as shown in Fig. 4 This makes the transactions immutable and improve security.

\section{IMPLEMENTATION}

In order to develop the smart e-commerce application the visual studio 2017 has to be installed. Visual Studio 2017 offers a brand new way of choosing the tool sets as shown in Fig.5.

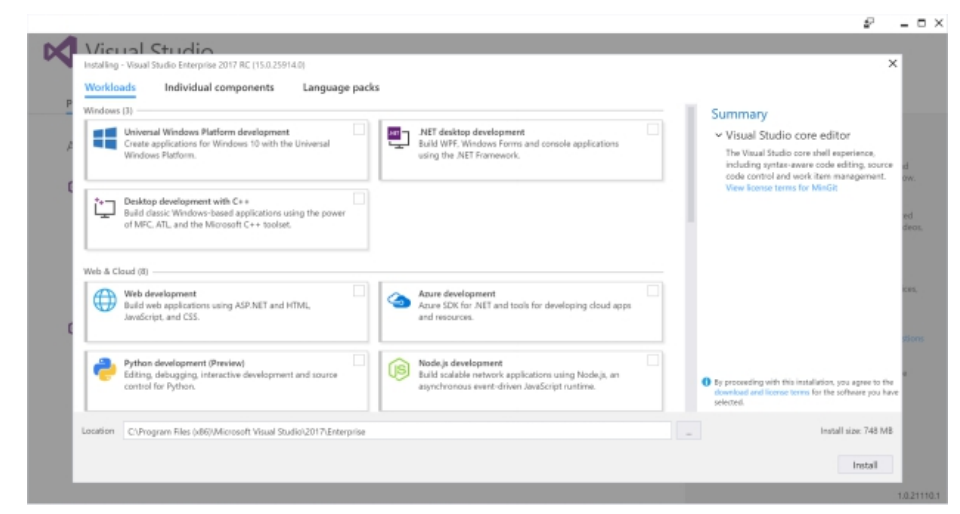

Figure 5: Visual Studio Tool Sets.

For this project web development need to be selected along with the asp.net core for web application and Xamarin for mobile application development from the Workloads section. After this click install and the Visual studio 2017 will be installed and will be ready to use.

\section{Sharp Proxy}

After installing the necessary tools the application has be run on a proxy server which is used to create a local environment. in order to keep the mobile application and web application in same environment. A proxy server is a web server that caches Internet resources for re-use by a set of client machines [22].In the below diagram the mobile and web application are running under common port 5000. In order to run the sharp proxy the sharp proxy server should be opened and sharpproxy.sin should be opened with visual studio then the start button on the top should be pressed in order to run the project. Then dialogue boxes appear where the port number you want the project to run on should be entered and click start. 


\section{IP Configuration}

Once the sharp proxy is running the other applications can be started. First the IP address has to configured in the web and mobile application. In the online Shopping folder open the sln file in administrator mode and run it with visual studio 2017 , then NewItemPage.xaml.sc file should be opened as shown in Fig.6 and the IP address should be configured and set to same as the IP address that appeared in the dialogue box when the sharp proxy was runned.

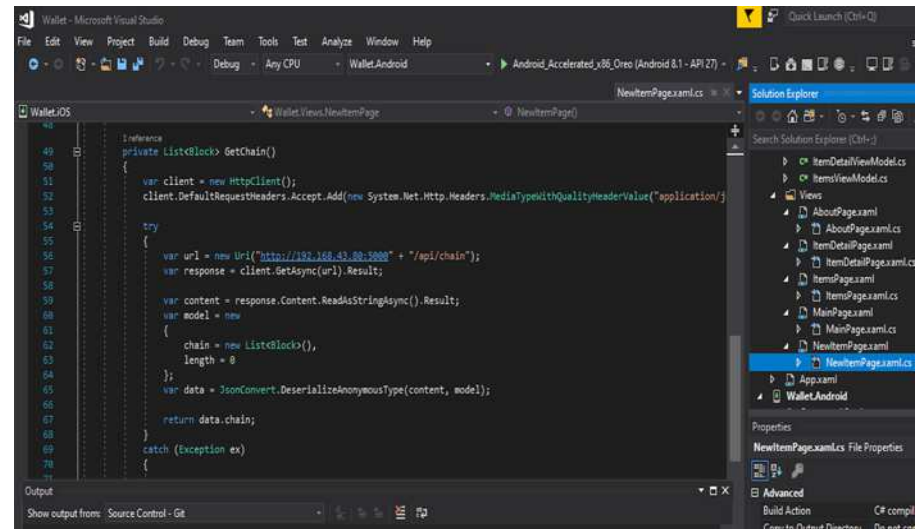

Figure 6: Web Application IP Configuration.

The IP configuration is needed here because whenever the network area is changed the IP changes so in order to execute the project the IP has to be set same in mobile wallet and the ecommerce site. After that vs file which is present in the online Shopping folder has to be opened then in the config file has top be opened with notepad. Then the IP address should also be configured there as shown in Fig. 7.

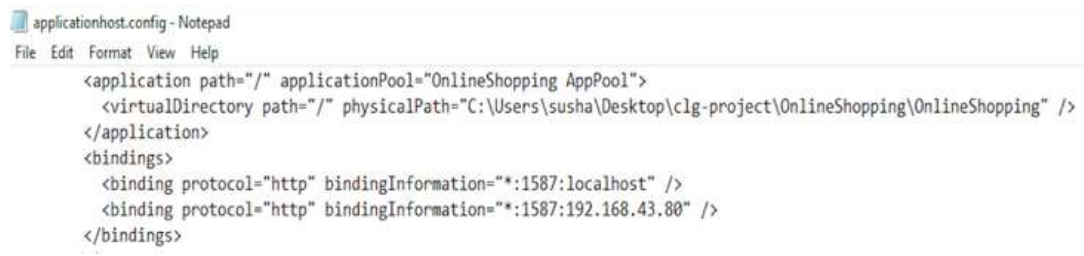

Figure 7: Web Application Config File.

Then the ip has to be configured in the mobile wallet. In order to configure this wallet application has to be opened with visual studio 2017 and first IP has to be configured in index.cshtml and set to same as the IP address that appeared in the dialogue box when the sharp proxy was runned as shown in Fig.8.

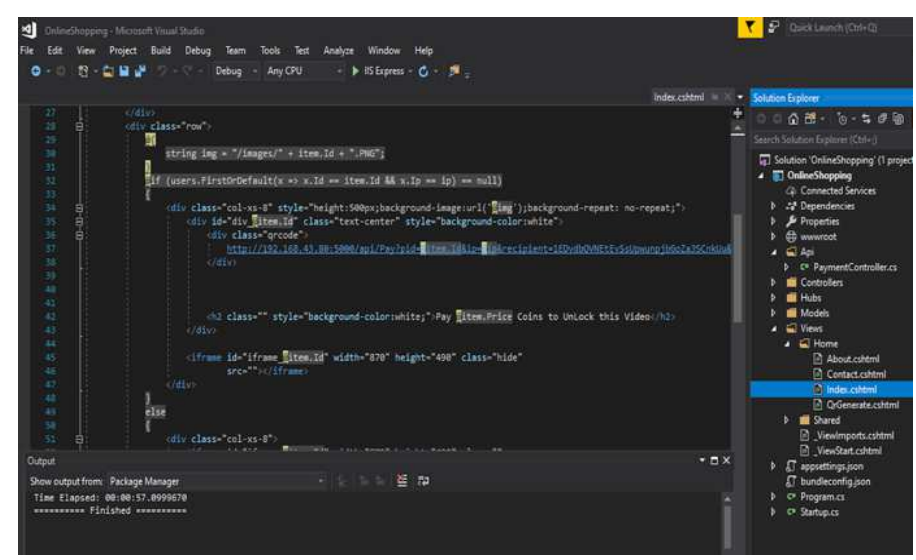

Figure 8: Mobile Application IP Configuration-1. 
Then the IP has to be configured in or Generate.cshtml file and set to same as the IP address that appeared in the dialogue box when the sharp proxy was runned as shown in Fig. 9.

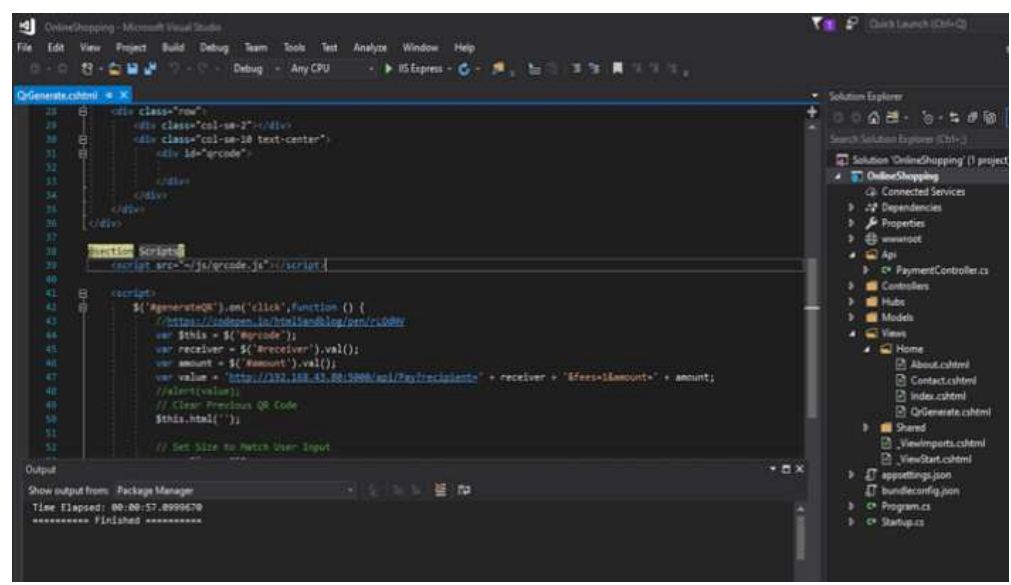

Figure 9: Mobile Application IP Configuration-2.

Once the IP configuration is done then the onlineShopping.sln and wallet.sln are runned followed by the blockchain. SIn file. In order to run web application first the sln files are opened with the visual studio and then start button or IIS express buttons are pressed on top. The orange bar appears on the bottom once the compilation process is completed and the web application gets automatically opened in the web browser.

In order to run the mobile application first the API level has to be set in wallet project buy opening AndroidManifest.xml file as shown in Fig. 10. Minimum android version is the API level that you want your application to run in an android phone with minimum specifications and Target android version is the is the maximum API level that a android device can have to run the application and. It has to be set as per the recent latest guide lines by Google play store to avoid errors.

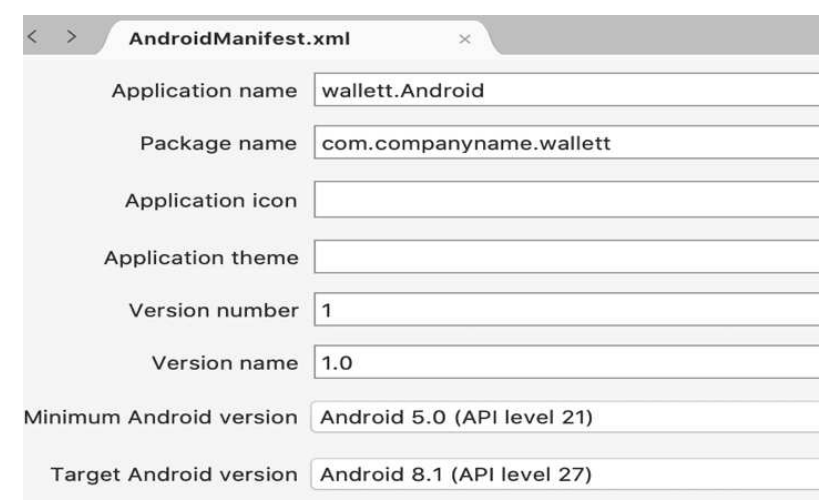

Figure 10: Application Program Interface Level.

Then the mobile has to be connected to the system using a USB cable and then the USB debugging options has to be enabled in order to deploy the mobile application in the actual physical mobile. To enable the USB debugging options, first setting in mobile have to opened and there build number as shown in Fig. 11 has to be tapped 7 times in order to get the developer options. After that in developer options USB debugging should be turned on. Once the USB debugging is enabled the system will detect the phone and the wallet application can be deployed to the mobile by clicking start option in the top. 


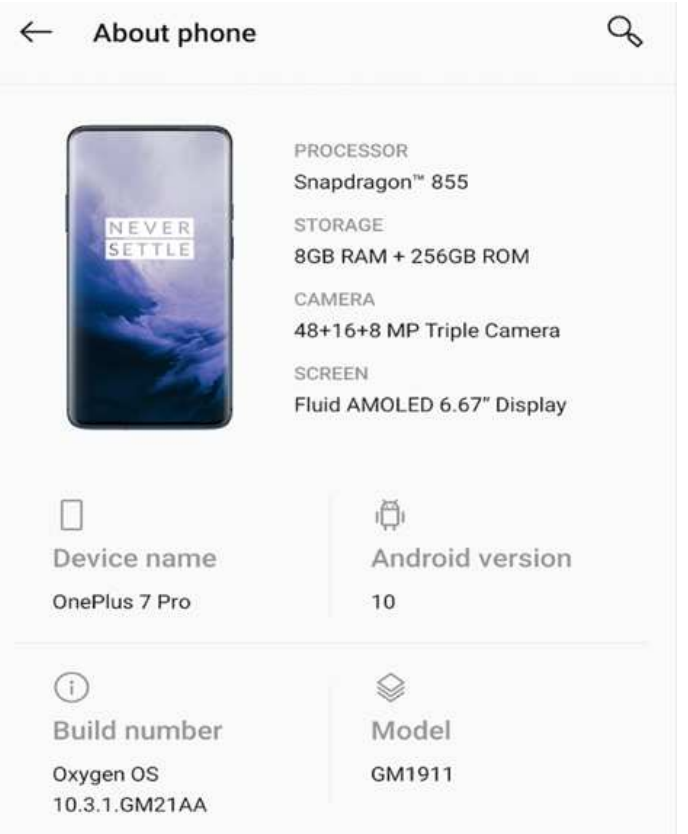

Figure 11: Build Number.

\section{RESULTS}

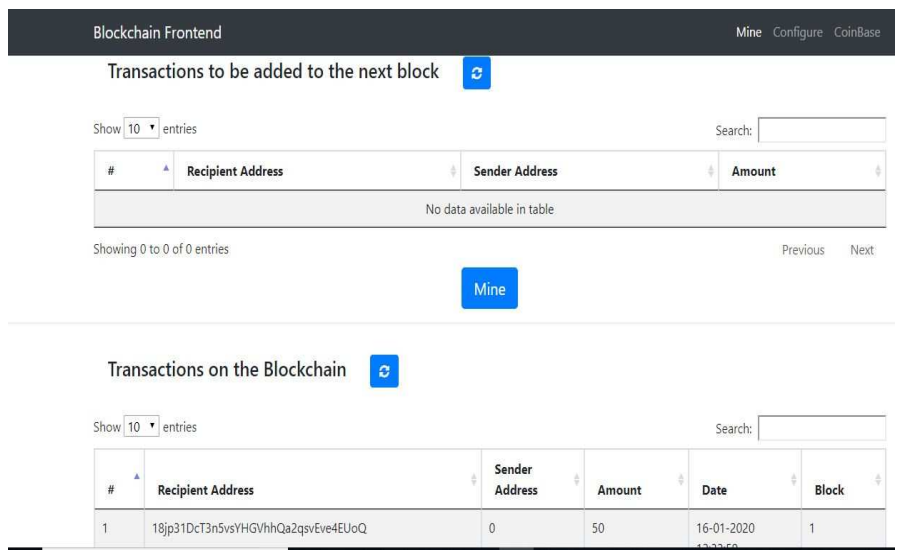

Figure 12: Mining New Transaction to a Block.

First in order to receive the coins to start of the applications first the user is given some coins i.e. 50 in this case and once the block is mined the transaction gets recorded in the blockchain. The mine button at the top is used to add the transactions inside the block as shown in Fig. 12. Here recipient represents the users address, sender is the system itself so it is represented with a zero, and amount is the amount sent by the system to user. This also consists of the information about when the transaction is mined with complete date and time along with the block number.

This entire part of the blockchain user interface is used to completely understand the main processes of the blockchain which the recording of the transactions, the process of mining and how the data gets stored.

The mining [23] here means the adding of transactions to the existing blockchain ledger of transactions distributed among all users of a blockchain and it also involves creating a hash block of transactions that cannot be easily forged, protecting the integrity of entire system of blockchain. 


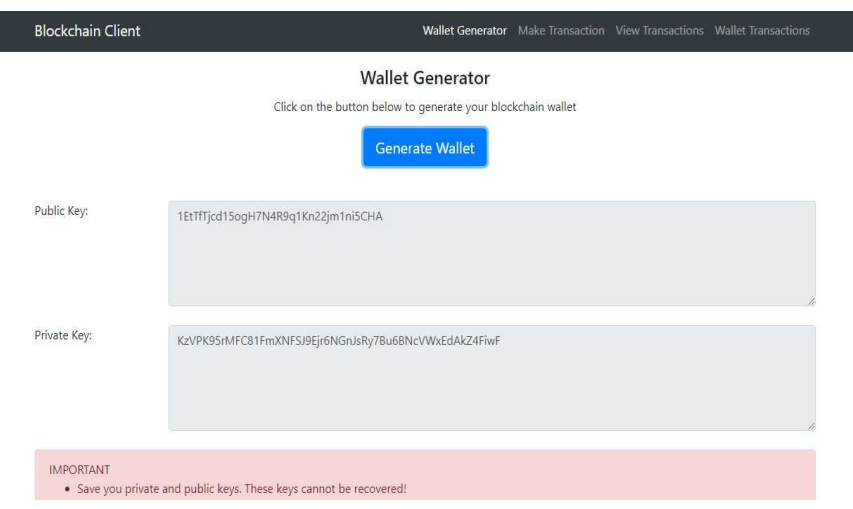

Figure 13: Wallet Generator.

Wallet generator is used to create crypto currency wallet which is unique for every user as shown in Fig. 13. It is basically used to generate public key and private key. Public key is shared among all as it is used to receive the assets and private key is kept secret and is used to send the assets. The generate wallet button at the top is used to generate a new wallet each time it is presses. In this particular project generate wallet is used in order to perform transactions between user and the ecommerce site.

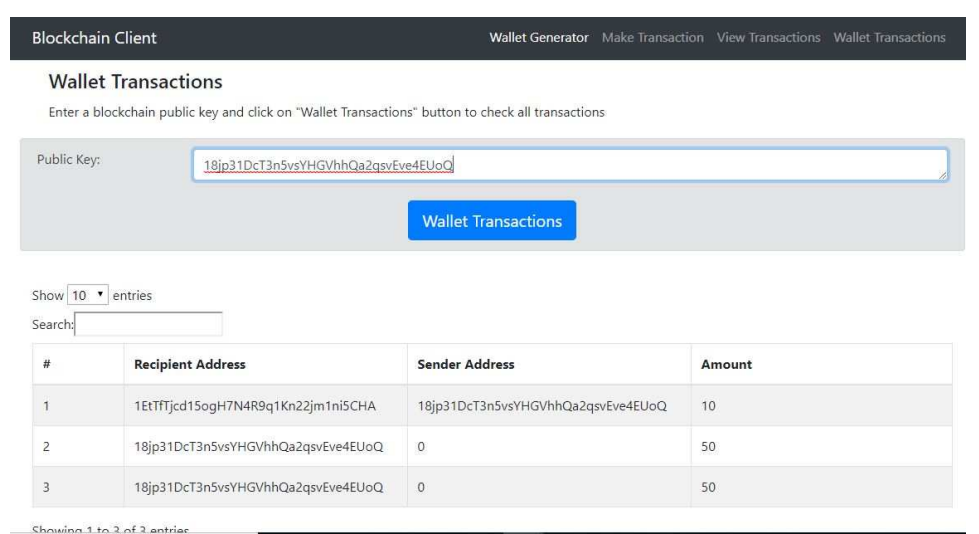

Figure 14: Wallet Transactions.

Wallet transactions page which is a part of blockchain UI is used to get all the transactions history done by a particular person from creation of the wallet till the current time as shown in Fig.14. In order to get the transaction details we just have to paste our public key and all transactions will be appeared. The recipient represents the person whom we sent the amount, sender represents the address of the sender and amount represents the total coins involved in transaction. This feature will enhance the transparency of the transactions and as the entire ledger is maintained there are less chances of fraud [24].

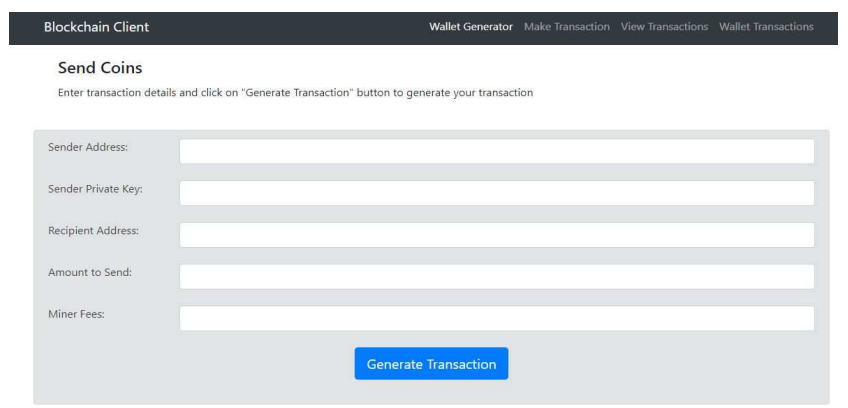

Figure 15: Transactions Generator. 
Generate transaction button is used to perform the transaction with the given credentials as shown in Fig, 15. The senders address means refers to person who wants to send certain amount of coins to the receiver. Senders private key means the key which is used to send the assets to another person and it should be kept secret. Miner fees represents the fees that we need to pay in order to perform every transaction and add that particular transaction in blockchain.

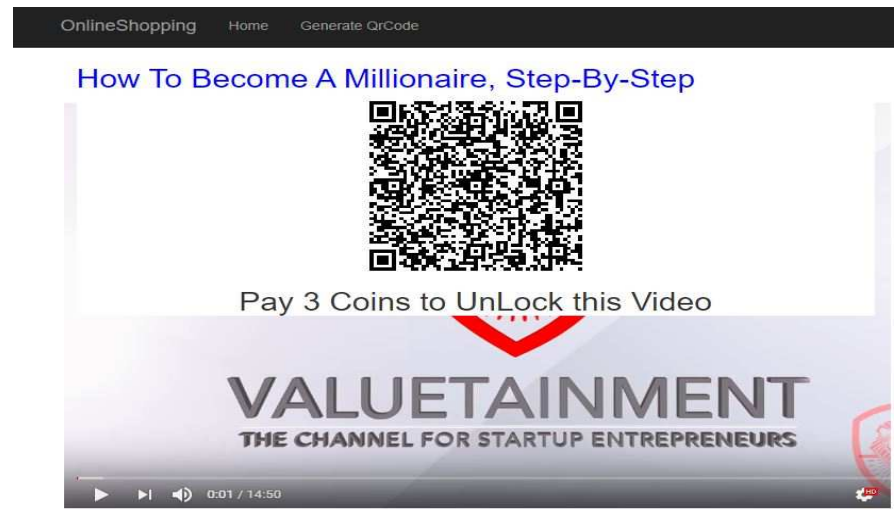

Figure 16: Video Unlocking Feature.

Fig. 16 represents the view of the digital content which is locked with the help of the QR code. This is the main part of the ecommerce site which involves the videos locked which can be unlocked using the QR code. This particular video as seen can be unlocked by sending three coins to the ecommerce site through QR code with help of the mobile wallet. Once the payment is made then the video will be unlocked and the digital content can be viewed.

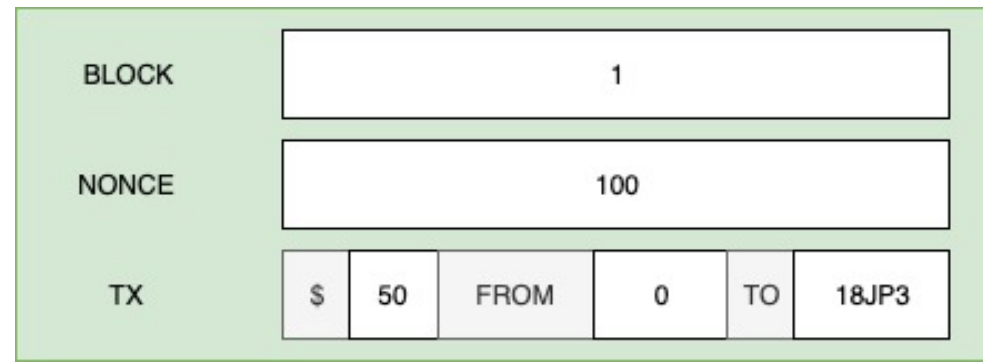

Figure 17: Storing of the Transaction in Blockchain.

Fig 17 is the representation of a block in the blockchain where the transaction is being stored. In this particular block which is the genesis block or the starting block a transaction of 50 dollars from system to the miner or the user is being represented and here the nonce [25] is used by miner to mine new blocks.

Mobile wallet as shown in Fig.18 is used to make the payments with help of QR code scanner. It consists of three windows. In the first window user has to enter the credentials which are private and public key and save the details in order to perform any transactions.

Second window which is the main window is used to make the payments by scanning the QR code. In this section you have to scan the QR code and enter the amount to send. After entering the details just click pay to proceed the transaction. It is app through which we will be able to scan the QR code and unlock the digital content.

Third window consists of the transaction information which includes total received coins, total number of coins deducted while sending and the remaining balance in the account. Apart from this the transactions ledger is also maintained. 


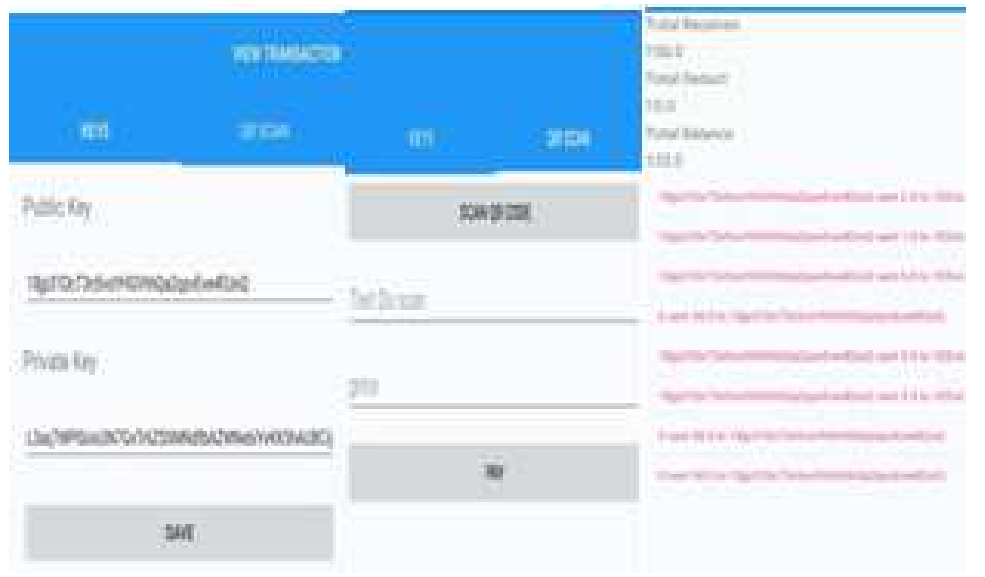

Figure 18: Mobile Wallet.

\section{CONCLUSIONS}

The use of the blockchain technology in the ecommerce improves security and reduces the frauds taking place thereby building the trust of the user. The storage of the transactions in the blockchain also adds more security as once entered cannot be changed. Also everything happening in the blockchain will be transparent. Not only ensures security but the speed of the payment system through crypto currency is very fast and accurate. By just scanning the destination address using the mobile wallet the transfer of the coins takes place. Also there is no need of carrying the credit card everywhere as mobile wallet will be sufficient for making payments.

\section{REFERENCES}

1. Nanehkaran, Y.A (2013). An introduction to electronic commerce. International journal of scientific \& technology research, 2(4). Retrievedfromhttp://www.ijstr.org/final-print/apr2013/An-Introduction-To-Electronic-Commerce.pdf.

2. Mohammed Ali, "How it takes 6 seconds to hack a credit card", proceedings of the academic IEEE security and privacy journal, 2016.

3. Azeemi, N. Zafar. "Delivering 4G (LTE) TO $5 G$ Migration with Supply Chain Management." International Journal of Electronics and Communication Engineering 6.1 (2017): 21-32.

4. V. Dudykevych, O. Bakay and Y. Lakh, "Investigation of Payment Cards systems information security control," 2013 IEEE 7th International Conference on Intelligent Data Acquisition and Advanced Computing Systems (IDAACS), Berlin, 2013, pp. 651654.

5. Apple Pay, https://www.apple.com/apple-pay/

6. Android Pay, https://www.android.com/pay/

7. S. Goyal, S. Yadav and M. Mathuria, "Exploring concept of QR code and its benefits in digital education system," 2016 International Conference on Advances in Computing, Communications and Informatics (ICACCI), Jaipur, 2016, pp. 11411147.

8. Ketaren, S. Otniel, et al. "Environmental health aspect in health emergency management (a case study: SinabungVulcanous Eruption)." Int. J. Appl. Nat. Sci. 5 (2016): 47-56.

9. S. Singh and N. Singh, "Blockchain: Future of financial and cyber security," 2nd International Conference on Contemporary Computing and Informatics (IC3I), pp. 463-467, 2016. 
10. C. Yang, Y. Chen, S. Chen and S. Wu, "A Reliable E-commerce Business Model Using Blockchain Based Product Grading System," 2019 IEEE 4th International Conference on Big Data Analytics (ICBDA), pp. 341-344, 2019.

11. Chavan, Amrita B., and K. Rajeswari. "The design and developement of decentralized digilocker using blockchain." International Journal of Computer Science Engineering and Information Technology Research (IJCSEITR) 9 (2019): 29-36.

12. Frey, Remo, Dominic Wörner, and Alexander Ilic. "Collaborative Filtering on the Blockchain: A Secure Recommender System for e-Commerce." Twenty-second Americas Conference on Information Systems (AMCIS), 2016.

13. Walport, M. G. C. S. A. "Distributed ledger technology: Beyond blockchain." UK Government Office for Science (2016).

14. A. B. Popa, I. M. Stan and R. Rughiniş, "Instant payment and latent transactions on the Ethereum Blockchain," 2018 17th RoEduNet Conference: Networking in Education and Research (RoEduNet),pp. 1-4, 2018.

15. K. Singh, N. Singh and D. Singh Kushwaha, "An Interoperable and Secure E-Wallet Architecture based on Digital Ledger Technology using Blockchain", International Conference on Computing, Power and Communication Technologies (GUCON), pp. 165-169, 2018.

16. Xingxiong Zhu, Qingsu He, ShanqiGuo. Application of block chain technology in supply chain finance [J]. China's circulation economy, 2018, 32 (03): 111-119.

17. Wadhwani, Priyanka, Akanksha Gaur, and Vipin Jain. "Cryptanalytic JH and Blake Hash Function for Authentication and Proposed Work Over Blake-512 on C Language." International Journal of Computer Science Engineering and Information Technology Research 4.3 (2014): 187-198.

18. M. C. Xenya and K. Quist-Aphetsi, "Decentralized Distributed Blockchain Ledger for Financial Transaction Backup Data," 2019 International Conference on Cyber Security and Internet of Things (ICSIoT), Accra, Ghana, 2019, pp. 34-36.

19. Satoshi Nakamoto, Bitcoin: A Peer-to-Peer Electronic Cash System, [online] Available: https://bitcoin.org/bitcoin.pdf.

20. Q. Liu and K. Li, "Decentration Transaction Method Based on Blockchain Technology," 2018 International Conference on Intelligent Transportation, Big Data \& Smart City (ICITBS), pp. 416-419, 2018.

21. S. Pahlajani, A. Kshirsagar and V. Pachghare, "Survey on Private Blockchain Consensus Algorithms," 2019 1st International Conference on Innovations in Information and Communication Technology (ICIICT), CHENNAI, India, 2019, pp. 1-6.

22. B. Lucas and R. V. Páez, "Consensus Algorithm for a Private Blockchain," 2019 IEEE 9th International Conference on Electronics Information and Emergency Communication (ICEIEC), Beijing, China, 2019, pp. 264-271.

23. Courtois and Nicolas, "The Longest Chain Rule and Programmed Self-Destruction of Crypto Currencies”, 2014

24. F. A. Masoud, D. H. Halabi and D. H. Halabi, "ASP.NET and JSP Frameworks in Model View Controller Implementation," 2006 2nd International Conference on Information \& Communication Technologies, Damascus, 2006, pp. 3593-3598.

25. Asp.net documentation https://docs.microsoft.com/enus/aspnet/core/?view=aspnetcore-2.2

26. C. L. Jeffery, S. R. Das and G. S. Bernal, "Proxy-sharing proxy servers," Proceedings of COM'96. First Annual Conference on Emerging Technologies and Applications in Communications, Portland, OR, USA, 1996, pp. 116-119.

27. S. Jiang and J. Wu, "Bitcoin Mining with Transaction Fees: A Game on the Block Size," 2019 IEEE International Conference on Blockchain (Blockchain), Atlanta, GA, USA, 2019, pp. 107-115.

28. Belonick, Paul, "Transparency is the New Privacy: Blockchain's Challenge for the Fourth Amendment”, 2019. 
29. D. Vujičić, D. Jagodić and S. Ranđić, "Blockchain technology, bitcoin, and Ethereum: A brief overview," 2018 17th International Symposium INFOTEH-JAHORINA (INFOTEH), East Sarajevo, pp. 1-6, 2018. 